\title{
Chronic Intramedullary Infusion of Prostaglandin E2 Stimulates Bone Formation Both in the Bone Marrow and in the Periosteum
}

\author{
MASAHIKo TAKADA, ITSUO YAMAMOTO, ITSUAKI YUU, YUSUKE KIGAMI, \\ YASUO OHNAKA, AND RIKUSHI MORITA \\ Department of Radiology, Shiga University of Medical Science, Shiga 520-21, Japan
}

\begin{abstract}
Prostaglandin E2 (PGE2) has a potent bone resorbing activity in vitro, but some recent studies have shown that PGE2 stimulates bone formation in vivo. The effects of PGE2 on the bone are therefore still controversial. We attempted to reveal the effects of PGE2 on bone in vivo more directly; we injected PGE2 continuously into the bone marrow and onto the periosteum and examined the local effects of PGE2 histologically or by bone densitometry. Following PGE2 infusion into the bone marrow, new bone was formed in the bone marrow around the infused site and following PGE2 infusion onto the periosteum, extensive periosteal bone formation was observed. Bone mineral content was also increased significantly in the PGE2 infused bones. The administration of cyclic AMP did not mimic the effects of PGE2. In contrast to in vitro experiments, the in vivo effect of PGE2 is predominantly to produce bone.
\end{abstract}

Key words: Prostaglandin E2, Bone mineral content, Bone formation, Periosteal reaction

(Endocrine Journal 42: 323-330, 1995)

PROSTAGLANDINS (PGs) have been shown to play an important role in bone remodeling [1]. It is well known that prostaglandin E (PGE) has a potent bone resorbing activity in vitro. PGE was a candidate for bone resorbing factors in rheumatoid arthritis, cancer-mediated hypercalcemia and osteolysis of bone metastasis, but several lines of evidence show that PGE stimulates bone formation in vivo: the systemic administration of prostaglandins, such as the daily subcutaneous injection of prostaglandin E2 (PGE2) [2-10] and a slow release pellet containing prostaglandin D2 [11], stimulated bone formation. The role of prostaglandins on the bone therefore remains to be clarified. To reveal the in vivo effects of PGE2 on the bone more directly, we used the method in which PGE2 is continuously injected into the bone

Received: November 4, 1994

Accepted: December 15, 1994

Correspondence to: Dr. Masahiko TAKADA, Department of Radiology, Shiga University of Medical Science, SetaTsukinowa-cho, Otsu, Shiga, 520-21, Japan marrow and onto the periosteum in rats by means of osmotic mini-pumps, and examined the effects of PGE2 histologically and in bone mineral measurements. Our results confirmed an anabolic effect of PGE2 on bone in vivo.

\section{Material and Methods}

\section{Materials}

PGE2 and 8-bromo-cAMP (8-B-cAMP) were purchased from Sasaki Chemical Co., Ltd. (Kyoto, Japan). PGE2 was dissolved in ethanol and diluted in phosphate buffered saline (PBS; pH 7.4) with $1 \%$ heat-inactivated bovine serum albumin (dilution buffer) for use. 8-bromo-cAMP was diluted in dilution buffer for use.

\section{Infusion in Rats}

10-12 month-old female Wistar rats (SPF; 270- 
290 g) were obtained from Shimizu Animal Supply Co. (Kyoto, Japan) and housed in light- and temperature-controlled rooms $\left(20-24{ }^{\circ} \mathrm{C}\right.$ and $12 \mathrm{~h} /$ $12 \mathrm{~h}$ light-dark cycle) under sanitary conditions. During the experiment periods, each animal was housed in a separate plastic cage and allowed free access to water and a $0.5 \%$ calcium diet. The animals were maintained under the guidelines established by the Animal Care Advisory Committee of Shiga University of Medical Science. PGE2 (10 to $100 \mu \mathrm{g}$ ) or vehicle alone was dissolved in $10 \mu l$ of $90 \%$ ethanol and diluted in dilution buffer and infused into the left femur with an Alzet mini-osmotic pump (model 2002, the mean filling volume was $213 \mu l$; Alza, Palo Alto, CA). In case. of intramedullary infusion, a hole $0.9 \mathrm{~mm}$ in diameter was made in the left femoral shaft with a motor-driven drill, and the $3 \mathrm{~mm}$ long tip of a 21 gauge stainless steel needle was inserted in such a way that the tip of needle was located in the center of the bone marrow. In the case of infusion onto the periosteum, the $5 \mathrm{~mm}$ long tip of a 21 gauge stainless steel needle was attached to the periosteum of the mid-femoral shaft. The needle tip was fixed to the bone with a fine thread and connected to the mini-pump with a sterile polyethylene tube. The mini-pump was implanted subcutaneously on the back and the infusion was continued for two weeks at an infusion rate of 0.5 $\mu l / h$, unless otherwise specified. In some experiments, 8-B-cAMP was also infused after dilution to a concentration of $25 \mathrm{mg} /$ pump. Three days prior to the end of the infusion, Calcein $(6 \mathrm{mg} / \mathrm{a}$ rat; Sigma Co., St. Louis) was injected subcutaneously for later fluorescent microscopic examination. In each group described above, at least six rats were examined.

\section{$X$-ray photographs, bone mineral assessment and histological examination}

After the infusion, the femur was excised and examined by means of soft X-ray photography, bone mineral measurement and histological study to investigate the effects of PGE2 infusion. The contra-lateral femur was also examined as a control. Soft X-ray photographs were taken at $31 \mathrm{KeV}$, $20 \mathrm{~mA} / \mathrm{s}$ with a Senomax X-ray apparatus (CGR, France). The untreated femur was always taken in the same film as the control. Bone mineral content
(BMC) was assessed by means of dual X-ray absorptiometry (DXA; Hologic QDR-2000, Waltham MA, fine mode with a pin-hole collimator). In preliminary experiments, the ash-weights of bones were measured after heating the bones at $600{ }^{\circ} \mathrm{C}$ for $24 \mathrm{~h}$ in an electric oven; ash-weight was compared with the results of DXA measurements. The correlation between ash-weight and BMC assessed by DXA was good $(\mathrm{y}=0.735 \mathrm{x}+7.969, \mathrm{r}=0.978, \mathrm{n}=15$, $P<0.001)$. Thus, in the following experiments, BMC was assessed by DXA, which showed an excellent reproducibility (C.V. of five separate measurements $=1.2 \%$ ). The contra-lateral intact femur was always studied as a control, and data were expressed as a ratio of the BMC of the infused bone to that of the intact bone in the same rat.

Finally, the femur was embedded in methyl methacrylate and sagittal sections ( $5 \mu \mathrm{m}$ thick) parallel to the long axis, extending from the proximal one-third to the distal one-third, were prepared. Some of these sections were stained by Goldner's method for microscopic examination and the others were used for the assessment of fluorescence labeling.

\section{Serum biochemical analysis}

At the end of the infusion, the rats were sacrificed by bleeding by cardiac puncture while anesthetized by an intra-peritoneal injection of sodium pentobarbital $(25 \mathrm{mg} / \mathrm{kg})$. Blood was saved for serum biochemical analysis. The serum calcium (Ca) concentration was measured by a colorimetric assay. Other biochemical parameters such as alkaline phosphatase and tartrate-resistant acid phosphatase were also assessed by colorimetric assays.

\section{Statistical analysis}

Statistical analysis was performed by unpaired $t$-test (Student's $t$ test). A $P$ value less than 0.05 was considered to be statistically significant.

\section{Results}

Intramedullary infusion

Figure 1 demonstrates the soft $\mathrm{X}$-ray photographs 
of the PGE2 infused femora. There was dose-dependent sclerosis around the inserted needle tip. A dose of $10 \mu \mathrm{g} /$ pump PGE2 (Fig. 1B) did not produce the sclerosis, while a dose of $100 \mu \mathrm{g} /$ pump PGE2 (Fig. 1C) always produced extensive bone sclerosis. In contrast, the infusion of dilution buffer alone and a dose of $25 \mathrm{mg} /$ pump 8-B-cAMP did not produce significant sclerosis (Fig. 1A, D).

The BMCs of PGE2-infused bones increased significantly (Fig. 2). The infusion of dilution buffer
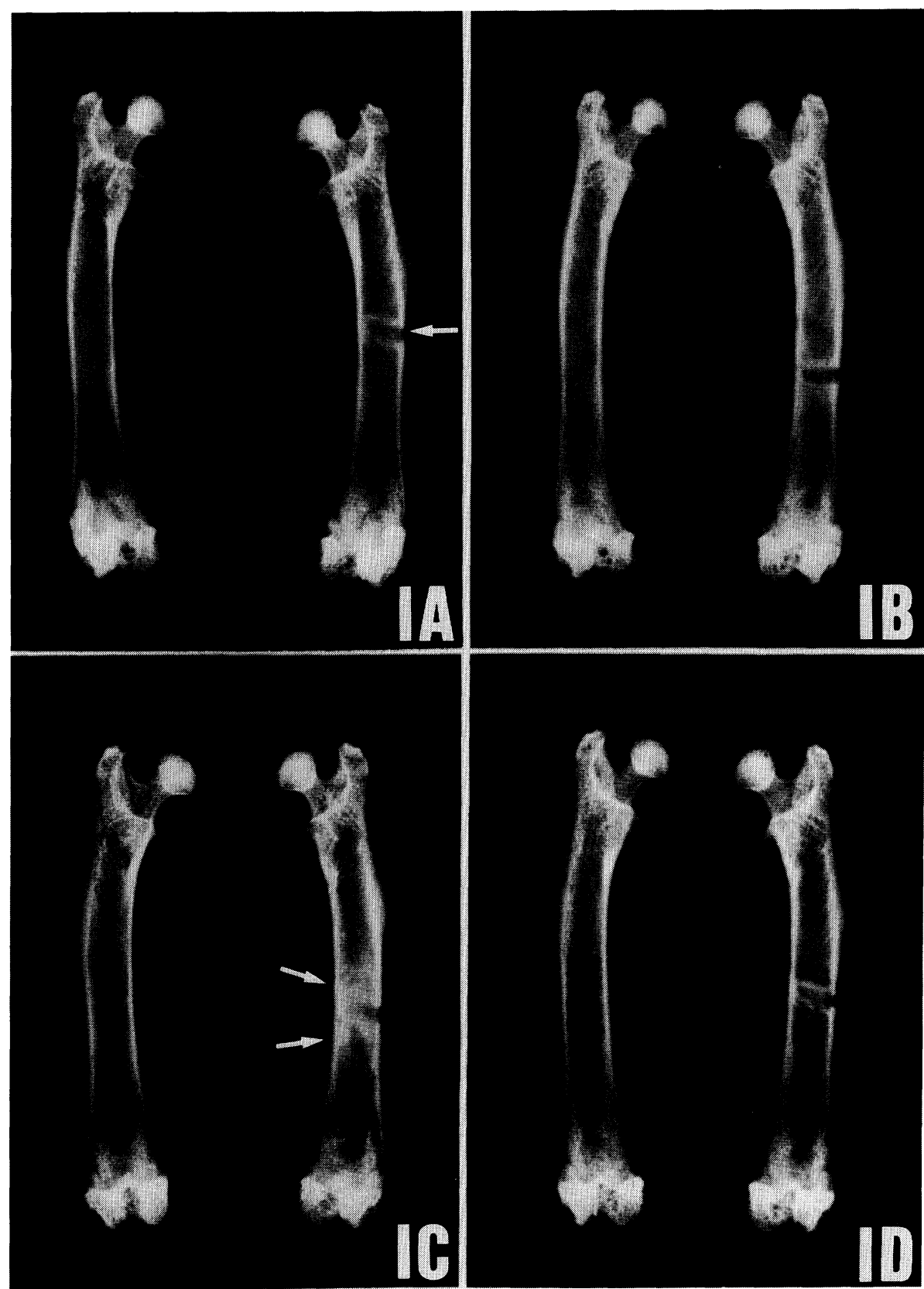

Fig. 1. Soft X-ray photographs of the intramedullary infused femora (Treated: right, Untreated: left). The dilution buffer alone (arrow shows the site of a needle inserted) (1A), $10 \mu \mathrm{g} /$ pump of PGE2 (1B) and $25 \mathrm{mg} /$ pump of 8-bromo-cyclic adenosine monophosphate (1D) did not produce significant sclerosis, but $100 \mu \mathrm{g} / \mathrm{pump}$ of PGE2 produced remarkable sclerosis around the inserted needle tip (arrows) (1C). Photographs show representatives of several similar findings. 


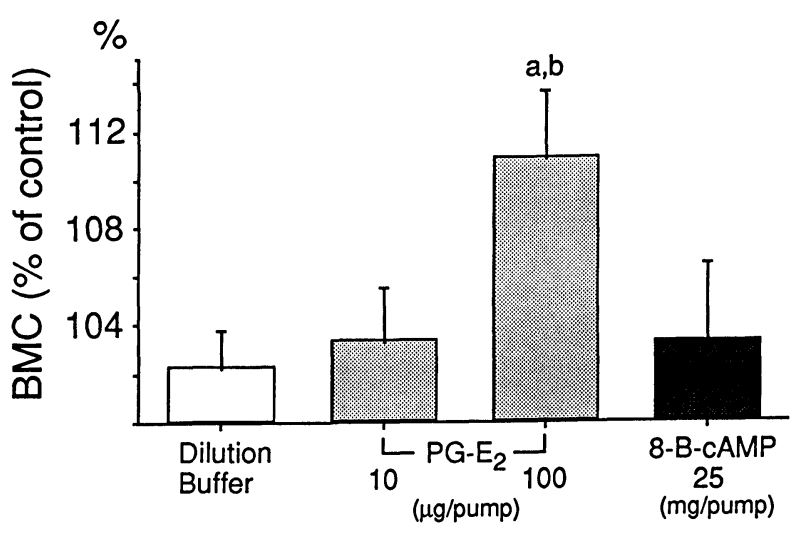

Fig. 2. BMCs of intramedullary infused femora. The infusion of dilution buffer alone, a low dose of PGE2 (10 $\mu \mathrm{g} /$ pump) and $25 \mathrm{mg} /$ pump of 8 -bromocyclic adenosine monophosphate caused a slight increase in the BMCs in the femora without significant differences among these three groups. The infusion of a high dose (100 $\mu \mathrm{g} /$ pump) of PGE2 increased BMC significantly compared with BMC in the contralateral intact femora (a: $P<0.001$ vs. vehicle, b: $P<0.001 v s .10 \mu \mathrm{g} /$ pump of PG-E2). Data are represented as the \% increase in $\mathrm{BMC}$ compared to the contralateral intact bone. The data shown are the means of samples (dilution buffer $n=12$, others $\mathrm{n}=6$ ) and bars represent the 1SD of the means.

alone or a low dose of PGE2 (10 $\mu \mathrm{g} /$ pump) caused a slight increase in the BMCs of the infused femora that was probably attributable to bone formation stimulated by the surgical procedure, without a significant difference between these two groups. The infusion of a high dose (100 $\mu \mathrm{g} / \mathrm{pump})$ of PGE2 caused a significant increase in BMC when compared to those of the contra-lateral intact femora $(P<0.001 v s$. control and $10 \mu \mathrm{g} /$ pump of PGE2), corresponding to the findings in soft $X$-ray photographs. Infusion of 8-B-cAMP ( $25 \mathrm{mg} /$ pump) did not cause an increase in $\mathrm{BMC}$, corresponding to the findings in soft $X$-ray photographs.

The histological findings are shown in Fig. 3. The infusion of dilution buffer alone produced proliferation of fibrous tissue around the infusion site. This was accompanied with a little bone formation in its periphery. There was also minimal periosteal bone formation (Fig. 3a). Infusion of PGE2 (100 $\mu \mathrm{g} /$ pump) produced remarkable intramedullary bone formation with extensive periosteal bone formation (Fig. 3b). In the bone marrow, small mononuclear cells replaced the fat tissue, which is usually found in normal bone marrow, and there appeared new bone extending from the inner surface of the cortex. Osteoid was covered by cuboid shaped osteoblasts around which proliferation of spindle shaped cells was observed (Fig. 3c). In addition, many small vessels were found near the newly formed bone (Fig. 3d). The infusion of dilution buffer alone and 8-B-cAMP (Fig. 3e) did not cause the bone marrow change. Strong Calcein labeling was found all over the surface of the new bone formed in the marrow (Fig. 3f).

\section{Infusion onto the periosteum}

Figure 4 shows a soft $X$-ray photograph of the PGE2-infused femora. There was a periosteal reaction on the mid-shaft of the femur (Fig. 4A), which was confirmed histologically (Fig. 4B). In addition, there appeared extensive new vessel formation sub-periosteally (Fig. 4C). In the both infusion methods, the contra-lateral femora did not show any histological changes in either the marrow or the periosteum at the concentration of PGE2 and 8-B-cAMP used (data not shown).

The intramedullary infusion of PGE2 and 8-BcAMP and the infusion of PGE2 on the periosteum caused significant changes neither in serum Ca levels, nor in other serum biochemical parameters levels such as alkaline phosphatase and tartrateresistant acid phosphatase (data not shown).

\section{Discussion}

PGE2 mediates the actions of several cytokines such as interleukin- 1 and tumor necrosis factor and has been proven to have a potent bone resorbing activity in vitro [15-18]. Recent studies show, however, that PGE2 stimulates bone formation in vivo. The subcutaneous infusion and oral administration of PGE2 increased the number of osteoblasts $[2,19]$ and a pellet slowly releasing prostaglandin E1 produced bone formation near the pellet [20]. The purpose of our study was to prove the effect of PGE2 on the bone more directly and to know the mechanism of the action of PGE2. For this purpose we performed direct intramedullary infusion of PGE2, which should show more directly the action of PGE2 than systemic infusion, in the adult rat femur, and examined the effects in soft 

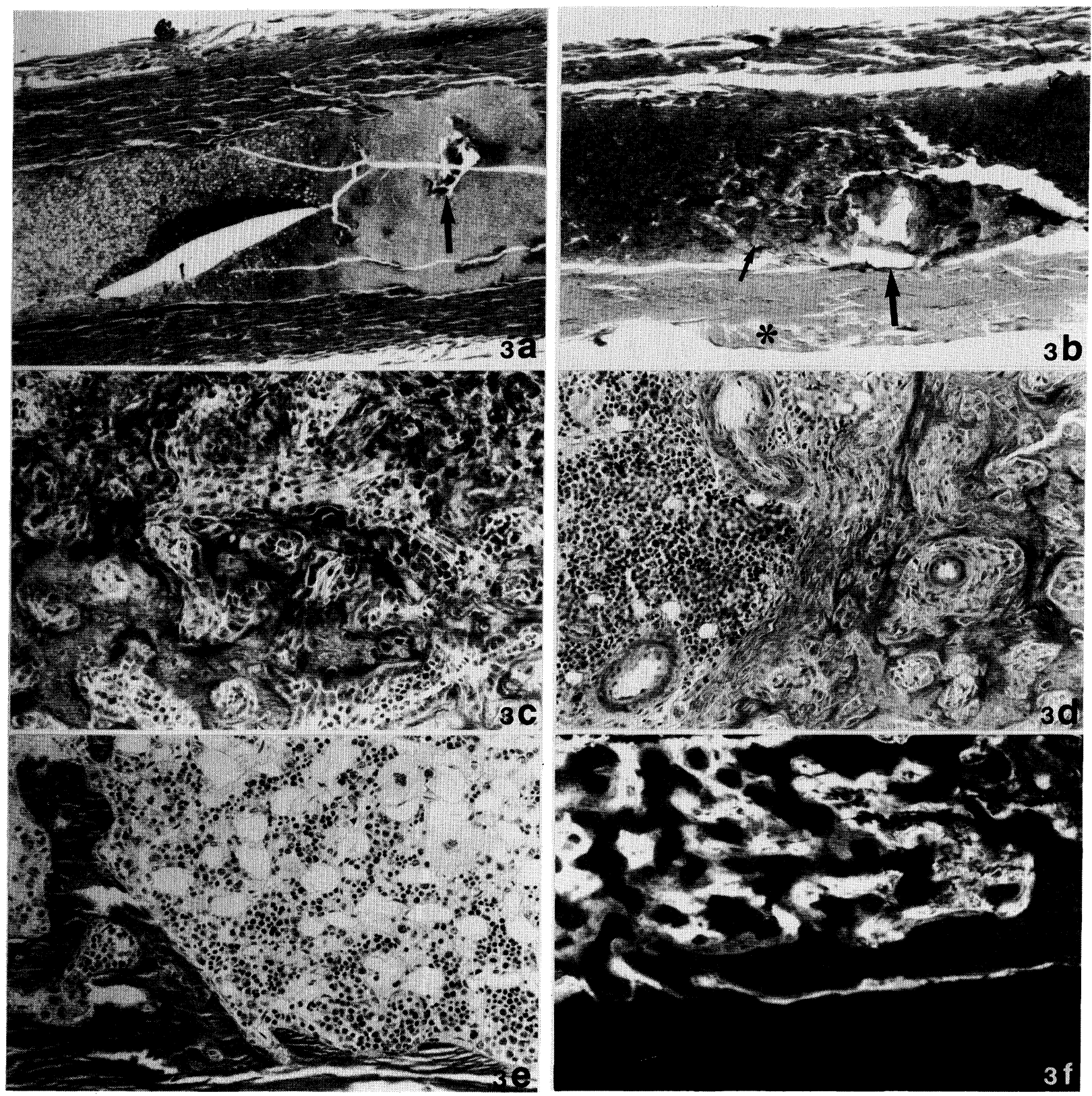

Fig. 3. Microscopic examination of intramedullary infused femora. The infusion of dilution buffer alone produced proliferation of fibrous tissue around the infusion site (arrow), accompanied by a little bone formation on its periphery and minimal periosteal bone formation (3a). The infusion of PGE2 (100 $\mu \mathrm{g} / \mathrm{pump})$ produced remarkable intramedullary bone formation with extensive periosteal bone formation (the large arrow shows the site of the needle tip, and the small arrows show bone formation in the marrow space. The star (*) shows the periosteal bone formation) ( $3 \mathrm{~b})$. Following the infusion of PGE2 (100 $\mu \mathrm{g} /$ pump), small mononuclear cells replaced the fat tissue and new bone was formed in the bone marrow. Osteoid was covered by cuboid shaped osteoblasts around which the proliferation of spindle shaped cells was observed (3c). In addition, many small vessels were found near the newly formed bone (3d). The infusion of 8 -bromo-cyclic adenosine monophosphate did not cause the bone marrow to change (3e). Following the infusion of PGE2 (100 $\mu \mathrm{g} / \mathrm{pump})$, strong Calcein labeling was observed all over the surface of the newly formed bone (3f). (magnification; $3 a, b: \times 5,3 c-f: \times 50$ ). 

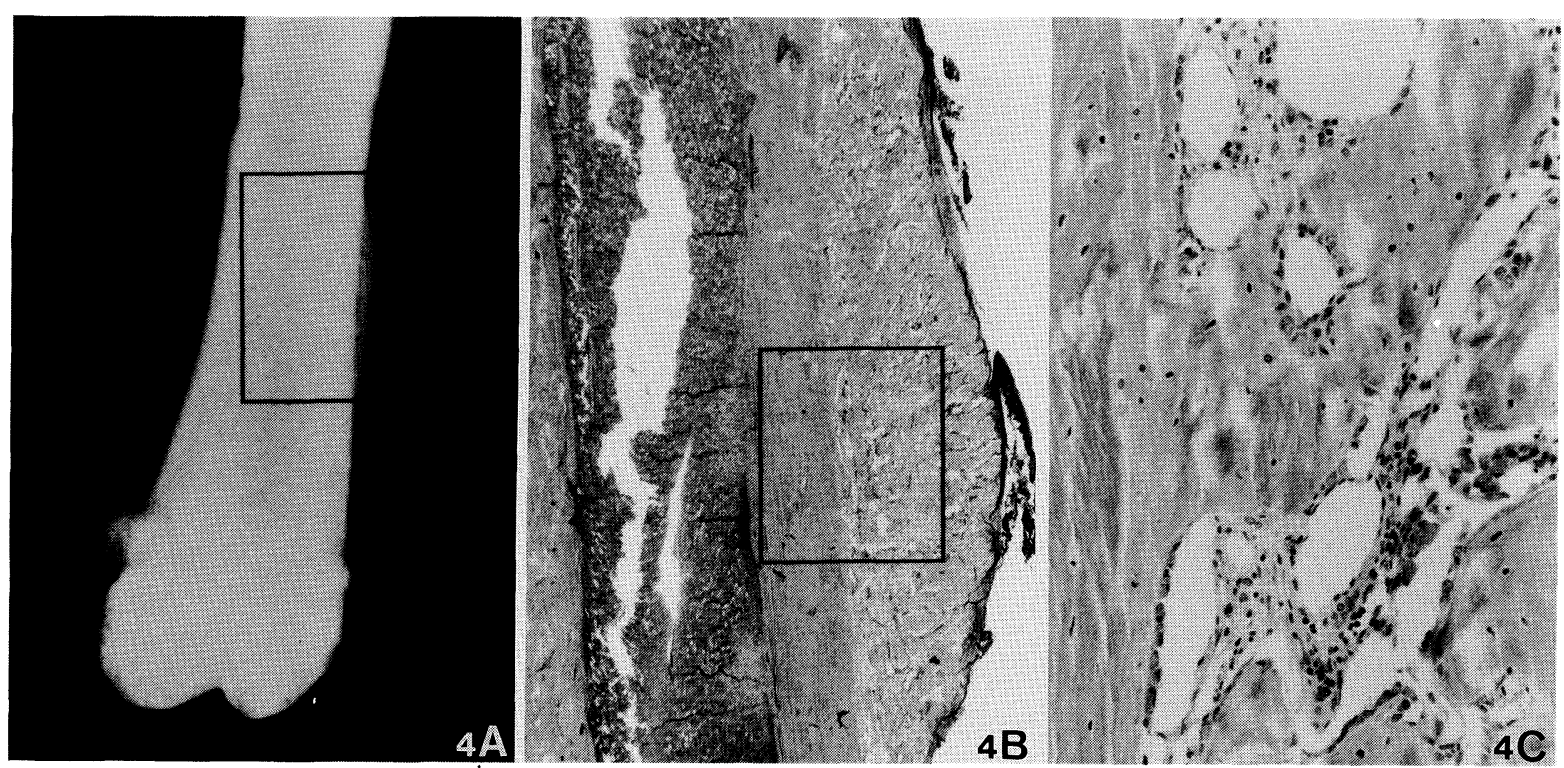

Fig. 4. The soft X-ray photograph and microscopic examination of femur following the infusion of PGE2 (100 $\mu \mathrm{g} / \mathrm{pump})$ onto the periosteum. Extensive periosteal bone formation on the mid-shaft of the femur is senn in the soft $X$-ray photograph $(4 \mathrm{~A})$. $4 \mathrm{~B}$ shows the histological finding of the periosteal bone formation, in the rectangle in $4 \mathrm{~A}$. In addition, there appeared to be extensive new vessel formation sub-periosteally $(4 \mathrm{C}$, the magnified view of the rectangle in $4 \mathrm{~B}$; magnification: $4 \mathrm{~B}: \times 5,4 \mathrm{C}: \times 50$ ).

X-ray photographs, bone mineral content measurement and histological sections.

Our study clearly demonstrated that PGE2 stimulated bone formation. By PGE2 infusion into the bone marrow, new bone was formed in the marrow around the infusion site (Fig. $3 b$ ) and BMC was increased at the infused sites. Periosteal infusion caused periosteal bone formation, while vehicle alone did not. Histologically, stimulation of bone resorption by PGE2 was not extensively observed. This might be attributable to the experimental periods in this study: activated osteoclasts may at first disappear during two weeks' infusion. In our experimental model, this was difficult to prove, because of concomitant effects of a surgical procedure early in the experiments. At least it is safe to say that chronic infusion of PGE2 results predominantly in bone formation.

We observed prominent new vessel formation around the new bone or rather new bone formation appeared around the newly formed vessels (Fig. 3d). Prostaglandin-E1 increased the intracortical remodeling space associated with increased vascularization [20]. Prostaglandin-E series are known to have actions on the vascular tissue [21$24]$, and thus the effects on the bone formation might be related to its action on the vascular system.

PGE2 is a well-known activator of adenylate cyclase in many cells including osteoblastic cells [25, 26]. We therefore infused 8-B-cAMP to mimic the action of PGE2, and no significant effects of cAMP analog were observed. Recent findings show the presence of three subtypes (EP1, EP2, and EP3) of PGE2 receptors, presumed coupled to stimulation of phospholipase $\mathrm{C}$, and stimulation and inhibition of adenylate cyclase [27]. In relation to the action on the bone, which subtype(s) of receptor is mainly involved is not certain. Our findings suggest that stimulation of adenylate cyclase may not be involved in the bone forming activity of PGE2. Other evidence supporting this hypothesis is that prostaglandin D2 stimulates calcification of human osteoblastic cells in vitro without the mediation of cAMP [28]. The mechanism of action of PGE2 on bone formation remains to be investigated.

PGE2 stimulated bone formation not only in the histological examination but also in X-ray photographs and in BMC measurement. PGE2 increased $\mathrm{BMC}$ in the infused bone. Since new bone is formed in the bone marrow without prior bone trabeculae, the injection of PGE2 into the bone mar- 
row would have a therapeutic potential in patients with senile osteoporosis, in which trabecular loss or discontinuity is thought to hamper the therapeutic responses due to various drugs. Local administration of PGE2 in our experiments did not cause side effects in rats.

In conclusion, we have shown the direct stimulation of bone formation of PGE2.

\section{Acknowledgments}

This work was supported in part by Grant in Aid for Scientific Research No. 05770679 from the Ministry of Education, Science and Culture of Japan. We express our thanks to technicians in the Toxicology Section, Hikone Research Laboratories, Maruho Co., Ltd. in Japan for making the histological sections.

\section{References}

1. Nordin RW, Jee WSS, High WB (1990) The role of prostaglandins in bone in vivo. Prostaglandins Leukot Essent Fatty Acids 41: 139-149.

2. Jee WSS, Mori S, Li XJ, Chan S (1990) Prostaglandin E2 enhances cortical bone mass and activates intracortical bone remodeling in intact and ovariectomized female rats. Bone 11: 253-266.

3. Jee WSS, Ke HZ, Li XJ (1991) Long-term anabolic effects of prostaglandin-E2 on tibial diaphyseal bone in male rats. Bone Miner 15: 33-55.

4. Jee WSS, Ke HZ, Li XJ (1992) Loss of prostaglandin E2-induced extra cortical bone after its withdrawal in rats. Bone Miner 17: 31-47.

5. Jee WSS, Akamine T, Ke HZ, Li XJ, Tang LY, Zeng QQ (1992) Prostaglandin E2 prevents disuse-induced cortical bone loss. Bone 13: 153-159.

6. Mori S, Jee WSS, Li XJ, Chan S, Kimmel, DB (1990) Effects of prostaglandin E2 on production of new cancellous bone in the axial skeleton of ovariectomized rats. Bone 11: 103-113.

7. Mori S, Jee WSS, Li XJ (1992) Production of new trabecular bone in osteopenic ovariectomized rats by prostaglandin E2. Calcif Tissue Int 50: 80-87.

8. Akamine T, Jee WSS, Ke HZ, Li XJ, Lin BY (1992) Prostaglandin E2 prevents bone loss and adds extra bone to immobilized distal femoral metaphysis in female rats. Bone 13: 11-22.

9. Ke HZ, Li M, Jee WSS (1992) Prostaglandin E2 prevents ovariectomy-induced cancellous bone loss in rats. Bone Miner 19: 45-62.

10. Ke HZ, Jee WSS, Mori S, Li XJ, Kimmel DB (1992) Effects of long-term daily administration of prostaglandin-E2 on maintaining elevated proximal tibial metaphyseal cancellous bone mass in male rats. Calcif Tissue Int 50: 245-252.

11. Takagi T, Yamamoto T, Asano S, Tamaki H (1993) Effect of prostaglandin D2 on the femoral bone mineral density in ovariectomized rats. Calcif Tissue Int 52: 442-446.

12. Chase LR, Aurbach GD (1970) The effect of parathyroid hormone on the concentration of adenosine $3^{\prime}, 5^{\prime}$ monophosphate in skeletal tissue in vitro. I Biol Chem 245: 1520-1526.

13. Marcus R, Orner FB (1979) Cyclic AMP production in rat calvaria in vitro: Interaction of prostaglandins with parathyroid hormone. Endocrinology 101: 1570-1578.

14. Klein NJ, Bowers PN, Raisz LG (1990) Evidence that adenosine $3^{\prime}$, 5'-monophosphate mediates hormonal stimulation of prostaglandin production in cultured mouse parietal bones. Endocrinology 126: 1070-1075.

15. O'Keefe RJ, Crabb ID, Puzas JE, Rosier RN (1992) Influence of prostaglandins on DNA and matrix synthesis in growth plate chondrocytes. J Bone Miner Res 7: 397-404.

16. Tashjian AH, Voelkel EF, Lazzaro M, Goad D, Bosma T, Levine L (1987) Tumor necrosis factor a (cachectin) stimulates bone resorption in mouse calvaira via a prostaglandin-mediated mechanism. Endocrinology 120: 2029-2036.

17. Akatsu T, Takahashi N, Udagawa N, Imamura K, Yamaguchi A, Sato K, Nagata N, Suda T (1991) Role of prostaglandins in interleukin-1-induced bone resorption in mice in vitro. J Bone Miner Res 6: 183-189.

18. Linkhart TA, MacCharles DC (1992) Interleukin-1 stimulates release of insulin-like growth factor-I from neonatal mouse calvaria by a prostaglandin synthesis-dependent mechanism. Endocrinology 131: 2297-2305.

19. Jee WSS, Ueno K, Kimmel DB, Woodbury DM, Price P, Woodbury LA (1987) The role of bone cells in increasing metaphyseal hard tissue in rapidly growing rats treated with prostaglandin E2. Bone 8: 171-178.

20. Miller SC, Marks Jr SC (1993) Local stimulation of new bone formation by prostaglandin E1: Quantitative histomorphometry and comparison of delivery by mini-pumps and controlled-release pellets. Bone 14: 143-151.

21. Johnston HH, Herzog JP, Lauler DP (1967) Effect of 
prostaglandin E1 on renal haemodynamics sodium and water excretion. Am J Physiol 213: 939-946.

22. Weiner R, Kaley G (1969) Influence of prostaglandin E1 on the terminal vascular beds. Am J Physiol 217: 563.

23. Fine LH, Trizna W (1977) Influence of prostaglandins on sodium transport of isolated medullary nephron segments. Am J Physiol 232: F383-390.

24. Haylor J, Towers J (1982) Renal vasodilator activity of prostaglandin E2 in the rats anaesthetized with pentobarbitone. Br J Pharmmacol 76: 131-137.

25. McCarthy TL, Centrella M, Canalis E (1990) Cyclic AMP induces insulin-like growth factor I synthesis in osteoblast-enriched cultures. J Biol Chem 265:
15353-15356.

26. McCarthy TL, Centrella M, Raisz LG, Canalis E (1991) Prostaglandin E2 stimulates Insulin-like Growth Factor I synthesis in osteoblast-enriched cultures from fetal rat bone. Endocrinology 128: 2895-2900.

27. Sugimoto $Y$, Namba T, Honda A, Hayashi $Y$, Negishi M, Ichikawa A, Narumiya S (1992) Cloning and expression of a cDNA for mouse prostaglandin E receptor EP3 subtype. J Biol Chem 267: 6463-6466.

28. Koshihara Y, Kawamura M (1989) Prostaglandin D2 stimulates calcification of human osteoblastic cells. Biochem Biophys Res Commun 159: 1206-1212. 